\title{
Publisher Correction: Unusual 4H-phase twinned noble metal nanokites
}

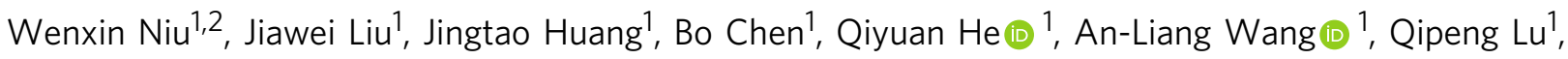
Ye Chen (1) ${ }^{1}$, Qinbai Yun ${ }^{1}$, Jie Wang ${ }^{1}$, Cuiling Li ${ }^{1}$, Ying Huang ${ }^{1}$, Zhuangchai Lai (1) ${ }^{1}$, Zhanxi Fan ${ }^{1}$, Xue-Jun Wu ${ }^{1} \&$ Hua Zhang (1) 1,3

Correction to: Nature Communications https://doi.org/10.1038/s41467-019-10764-2, published online 28 June 2019.

The original version of this Article contained an error in the author affiliations.

Affiliation 3 incorrectly read 'Department of Chemistry, City University of Hong Kong, Kowloon, Hong Kong.'

This has now been corrected in both the PDF and HTML versions of the Article.

Published online: 12 July 2019

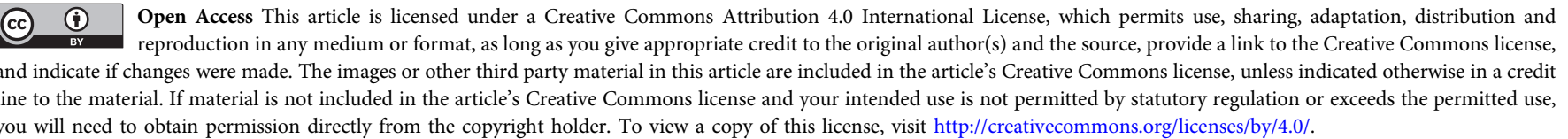

(C) The Author(s) 2019

\footnotetext{
${ }^{1}$ Center for Programmable Materials, School of Materials Science and Engineering, Nanyang Technological University, Singapore 639798, Singapore.

2 State Key Laboratory of Electroanalytical Chemistry, Changchun Institute of Applied Chemistry, Chinese Academy of Sciences, Changchun 130022, China. ${ }^{3}$ Department of Chemistry, City University of Hong Kong, Kowloon, Hong Kong, China. Correspondence and requests for materials should be addressed to H.Z. (email: hzhang@ntu.edu.sg or hua.zhang@cityu.edu.hk)
} 